\title{
Enseñanza de lenguas indígenas mediadas por las TIC en América Latina
}

\author{
Teaching indigenous languages using ICT in Latin America
}

\author{
María J. Ríos-Colmenárez ${ }^{a}$
}

\begin{abstract}
:
More than 500 indigenous groups are currently distributed in Latin America, they speak more than 400 different languages. In recent years, there have been several educational policies that have been implemented in most of the region in order to preserve, among other aspects, the indigenous languages and, consequently, the cultural heritage that identifies each particular ethnic group. Most of the specific policies that were previously mentioned are focused on the use of Information and Communication Technologies (ICT) as didactic means for teaching these specific languages in basic education in Latin American countries. This report describes the findings of a previous exploratory research that had as a purpose knowing the problems the teaching of indigenous languages using ICT in Latin America faces today. The information gathered from an online questionnaire aimed at teachers who teach indigenous languages in Latin America is reviewed and analyzed. Some recent educational proposals available on the Web are also described. Among the findings are the need of Internet access and the lack of digital skills of teachers and students. There is also evidence of the increase in multimedia applications for teaching indigenous languages which describe not only the languages per se but also the cultural aspects that characterize them. Due to this, it can be assumed a need for a reform of the instructional design including a view that favors the appropriation of knowledge and the worldview of the indigenous groups; that is to say, teaching indigenous languages beyond the literal translation of their contents and expressions.
\end{abstract}

\section{Keywords:}

Indigenous languages, ICT, Latin America, educational policies, indigenous worldview

\section{Resumen:}

Más de 500 pueblos indígenas se distribuyen actualmente en América Latina con el empleo de más de 400 lenguas distintas. En los últimos años han sido varias las políticas educativas que se han implementado en gran parte de la región a fin de preservar, entre otros aspectos, el idioma indígena, y con ello el acervo cultural que identifica a cada étnia en particular. Entre las mencionadas políticas muchas se han orientado al uso de las Tecnologías de la Información y la Comunicación (TIC) como medios didácticos para la enseñanza de estas lenguas especialmente en Educación Básica en países de América Latina. El presente reporte da cuenta de una indagación exploratoria previa con el propósito de conocer los problemas que afronta hoy la enseñanza de las lenguas indígenas mediadas por las TIC en América Latina. Se revisa y analiza la información obtenida de un cuestionario en línea dirigido a docentes que enseñan lenguas indígenas en América Latina, y se describen algunas propuestas educativas recientes disponibles en la Web. Entre los hallazgos se destacan requerimientos como: acceso a Internet y fortalecimiento de las habilidades digitales de docentes y estudiantes, también se evidencia el incremento de aplicaciones multimedia para la enseñanza en lengua indígena encontrándose algunos aspectos que las caracterizan y que permiten suponer la necesidad de reformular el diseño instruccional de las mismas con miras a favorecer la apropiación de los saberes y la cosmovisión de los pueblos originarios, más allá de la mera traducción literal de contenidos y expresiones propias de estas lenguas.

\section{Palabras clave:}

Lenguas indígenas, TIC, América Latina, políticas educativas, cosmovisión indígena 


\section{INTRODUCCIÓN}

Históricamente, América Latina se ha destacado por su diversidad lingüística y, por ende cultural. Si bien el idioma español es uno de los más difundidos en la región, no puede dejarse de lado la existencia de distintas variedades de este idioma y otros que incluso ya se empleaban antes de la llegada de los conquistadores europeos, como son las lenguas indígenas. Muchas de estas lenguas han desaparecido, estimándose que al menos la mitad de las que se hablaban en el territorio ya no existen, debido inicialmente al proceso de colonización, y luego, por aspectos como la extinción natural, efectos del español como idioma dominante, y la globalización, por solo mencionar algunos. Actualmente se calcula que la región latinoamericana cuenta con más de 400 lenguas diferentes habladas por unos 50 millones de indígenas, siendo México, Guatemala, Ecuador, Bolivia, Perú, Argentina, Brasil, Colombia y Chile, algunos de los países con presencia importante de número de nativos de estas lenguas (Godenzzi, 2001; Moreno, 2006). Caso excepcional ocurre en Paraguay, en el que un $90 \%$ de la población no indígena habla guaraní como segunda lengua.

Para los pueblos indígenas las lenguas representan símbolos de identidad y pertenencia a un grupo, vehículos de valores éticos $\mathrm{y}$, "sistemas de conocimientos mediante los cuales estos pueblos forman un todo con la tierra y son cruciales para su supervivencia" (Degawan, 2019). No obstante, se encuentran en riesgo de desaparición aproximadamente 200 lenguas indígenas en toda la extensión latinoamericana (Organización de las Naciones Unidas para la Educación, la Ciencia y la Cultura, UNESCO, 2010).

Lo anterior ha sido considerado en los últimos años en las diferentes políticas gubernamentales de la región con miras a preservar, entre otros aspectos, el idioma indígena, y con ello el acervo cultural que identifica a cada étnia en particular. Así, en concordancia, con distintas iniciativas como la Agenda 2030 para el Desarrollo Sostenible adoptada por la Organización de las Naciones Unidas (ONU), específicamente en el Objetivo 4 y el Marco de Acción para su realización, se hace referencia al compromiso internacional de sus países miembros a brindar una educación de calidad, inclusiva y equitativa, así como también, ofrecer oportunidades de aprendizaje útiles para la vida a todas las personas y grupos vulnerables, entre los cuales se encuentran los pueblos indígenas. (ONU, 2015).

De igual manera, las políticas atienden lo establecido en la Declaración de las Naciones Unidas sobre los Derechos de los Pueblos Indígenas en procura de la revitalización, uso, fomento y transmisión de sus lenguas, tradiciones orales, escritura y cosmovisiones. A ello se une, además, el Convenio
169 de la Organización Internacional del Trabajo, en donde se declara el compromiso de garantizar el derecho de los pueblos indígenas a establecer sus sistemas educativos, así como proteger y revitalizar sus lenguas y culturas, incluyendo su historia, conocimientos y sistemas de valores en la educación considerando sus propias aspiraciones sociales, económicas y culturales.

En ese orden de ideas, países como el Estado Plurinacional de Bolivia, Ecuador, México, Perú, Venezuela y Paraguay, reconocen en sus textos constitucionales la importancia de los derechos indígenas; ello, junto a otras leyes, decretos y acuerdos, como en el caso de Chile y Guatemala, fortalecen especialmente la relevancia de las lenguas indígenas en el ámbito educativo a través de diversas instituciones, programas y propuestas curriculares que procuran favorecer la educación intercultural y establecer un diálogo con los conocimientos de pueblos indígenas. (UNESCO, 2017).

En Bolivia se encuentra el Modelo educativo socio-productivo comunitario, la Ley 070 (2010) que hace alusión al Sistema educativo intracultural, intercultural y plurilingüe, así como también el Instituto Plurinacional de Lenguas y Culturas y, el Sistema Plurinacional de Maestros (Decreto 156 del año 2009). (Mercado, 2014).

En Chile, por su parte, se encuentra el Programa de Educación Intercultural Bilingüe, y organismos como la Secretaría de Educación Intercultural Indígena y, la Corporación Nacional de Desarrollo Indígena en áreas de Educación Intercultural Bilingüe para atender a nivel preescolar (Lagos, 2015).

Mientras que en Ecuador están la Subsecretaría de Educación Intercultural Bilingüe, el Instituto de Idiomas, Ciencias y Saberes Ancestrales y, el Modelo del Sistema de Educación Intercultural Bilingüe. (UNESCO, 2017)

Otro país es México, en donde se encuentra la Dirección General de Educación Indígena, el Instituto Nacional de Lenguas Indígenas y la Coordinación General de Educación Intercultural Bilingüe. Guatemala, por su lado, cuenta con la Dirección General de Educación Bilingüe Intercultural, el Viceministerio de Educación Bilingüe Intercultural y, la Academia de las Lenguas Mayas de Guatemala. Y, Perú con la Dirección Nacional de Educación Bilingüe Intercultural, la Dirección General de Educación Básica Alternativa, Intercultural, Bilingüe y de Servicios Educativos en el Ámbito Rural y, el Plan Nacional de Educación Intercultural Bilingüe al 2021. (UNESCO, 2017)

Pese a tales iniciativas en la región, tal como se expresó en el Foro Permanente para las cuestiones Indígenas (ONU,2019a), es necesario realizar mayores esfuerzos siendo la apropiación de todo tipo de recursos tecnológicos una de las demandas más 
fehacientes del actual contexto educativo para promover la mejor calidad y equidad de las instituciones escolares.

Así, dada la existencia de una gran diversidad de situaciones sociolingüísticas, se requiere "imaginar estrategias de educación intercultural diferenciadas que respondan a las características sociolingüísticas específicas de cada región, a las expectativas de su población y a las necesidades de aprendizaje de los educandos"; de allí que las Tecnologías de la Información y la Comunicación (TIC) pueden adquirir un papel significativo para la Educación Intercultural (Guerrero y Dote, 2011). Sin embargo, las medidas tomadas al respecto, muchas veces se limitan al apoyo económico para la realización de "grabaciones de idiomas, incluidas las transcripciones, traducciones y anotaciones de grabaciones audiovisuales, mientras que los programas de revitalización de las lenguas solo reciben fondos limitados" (ONU, 2019b).

De todo lo antes expuesto, se desprende el interés por indagar lo concerniente al uso de las TIC como estrategia para la enseñanza de lenguas indígenas en América Latina, proponiéndose, para ello, la revisión y análisis de la información obtenida de un cuestionario en línea dirigido a docentes que enseñan lenguas indígenas en la región, así como también la descripción de algunas propuestas educativas recientes disponibles en la Web.

\section{LA ENSEÑANZA EN LENGUAS INDÍGENAS}

En las últimas décadas ha cobrado especial relevancia la enseñanza de cualquier lengua concebida como un sistema de conocimiento íntimamente vinculado a aspectos de índole cultural, social e histórico, procurando no solo que se constituya en medio de comprensión, sino también que favorezca el conocimiento de la diversidad cultural de los pueblos y, en consecuencia, su identidad. En ese sentido, la competencia comunicativa relaciona la actividad lingüística con algunos aspectos de la comunidad hablante, al menos en tres campos: las referencias culturales, las rutinas y usos convencionales, y las convenciones sociales y comportamientos no verbales (dos Reis Batista, 2008).

De igual manera, para los propios hablantes, su lengua se constituye en una representación de su propia identidad, el vehículo de sus valores éticos y la pertenencia a un grupo. Así, las lenguas indígenas, como cualquier otra lengua, vienen a ser prácticas sociales construidas en atención a culturas históricas que se han caracterizado por contar con sus propias normas, sentidos y significados del mundo.

$\mathrm{Si}$ bien es cierto que en América Latina existe una gran diversidad de lenguas indígenas, no es posible negar que el español ha actuado por siglos como lengua dominante condicionando; en general, la realidad social de los hablantes de los pueblos originarios (lenguas dominadas) ha estado signada por el acceso a determinados roles sociales teniendo que abdicar forzosamente de su lengua propia (Rotaetxe, 1990). De ello no escapan, por ejemplo, los niños y niñas indígenas en edad escolar quienes aún cuando se encuentran amparados por la Convención sobre los Derechos del Niño de las Naciones Unidas (ONU, 1989) en cuanto al derecho que tienen a la educación y a aprender y usar el idioma de su familia, no dejan de presentar desventajas educativas relacionadas con la etnia y el idioma, debido a que la enseñanza es impartida en una lengua distinta de la materna.

En Perú para el año 2011, los niños y niñas hispanohablantes tenían más de siete veces más posibilidades que los hablantes de idiomas indígenas de alcanzar niveles satisfactorios en materia de lectura; en Guatemala, para el año 2006 de "los escolares pobres y hablantes de lenguas minoritarias (en su mayoría indígenas) en su hogar, solo el 38\% habían adquirido los elementos básicos de matemáticas, mientras que el $77 \%$ de los escolares ricos e hispanohablantes habían alcanzado ese nivel" (UNESCO, 2016, p.3).

Se plantea, en consecuencia, la necesidad de que los niños y niñas puedan hablar en su propia lengua en el contexto escolar, en el entendido que desde sus primeros años de vida, la lengua materna es el vehículo por el cual acceden al conocimiento. Si por el contrario, la escuela establece como "norma la adquisición rápida del español envía otro mensaje: las dificultades son responsabilidad del estudiante, no del modelo pedagógico o de su implementación” (Sordo, 2017).

Otra dificultad tiene que ver con los maestros cuya lengua materna es un idioma indígena, pero en muchos casos, como por ejemplo en México, no se encuentran del todo cualificados en comparación con otros docentes. A este respecto, cabe destacar que algunos países como Colombia, Guyana, Paraguay y Perú, han implementado reformas que pretenden ampliar el acceso a la educación y, la adaptación de los planes de estudios y de las prácticas pedagógicas a las necesidades de los niños y niñas indígenas. En Paraguay, especialmente, se ha hecho énfasis en la preparación de material educativo en diversos idiomas (UNESCO, 2016).

En ese orden de ideas, se requiere considerar el desarrollo de estrategias didácticas que respondan a las características sociolingüísticas y a las necesidades de aprendizaje de los educandos, pudiendo el uso de las Tecnologías de la Información y la Comunicación "ser de gran ayuda para desarrollar competencias lingüísticas dentro de un proceso de revaloración y resignificación de la cultura indígena". (Dote y Guerrero, 2011, p.1) 


\section{INTEGRACIÓN DE LAS TIC EN LA ENSEÑANZA DE LENGUAS INDÍGENAS}

Frente a las demandas del mundo globalizado del presente siglo, la Agenda digital para América Latina y el Caribe (eLAC2018), en su apartado "Cultura, inclusión y habilidades digitales", estableció la necesidad de "Masificar el acceso a servicios digitales y la producción y oferta de contenidos, asegurando la inclusión de toda la población, estimulando también la producción, la oferta y el uso de contenidos en lenguas indígenas y originarias” (Art. 18).

Desde el punto de vista educativo, la integración de las TIC en los procesos de enseñanza debe responder a la consecución de los objetivos curriculares y, por supuesto, motivar a los estudiantes hacia una mejor comprensión y construcción de su propio aprendizaje. No obstante, muchas veces "aprender con las TIC aparece como el foco de atención, sin un objetivo curricular de aprendizaje en mente" (Sánchez, 2008, p. 3), lo que concuerda con un enfoque tecnocéntrico de integración de dichas herramientas, ya que la mirada se centra en el manejo instrumental de la tecnología. Para el caso de las lenguas indígenas, las tecnologías pudieran ser útiles para el aprendizaje, la promoción, fortalecimiento, y difusión de las mismas.

Concretamente la enseñanza de las lenguas indígenas, en general, mantiene rasgos que tradicionalmente han estado presentes con o sin la incorporación de las TIC, que convergen en un proceso de apropiación de la cultura occidental y del idioma dominante, corriéndose el riesgo de estudiantes indígenas que pierden contacto directo con sus raíces ancestrales y minimizan sus interacciones, lo que afecta, a su vez, el normal desarrollo de la competencia lingüística de su propia lengua. (Hinton, 2001).

Especialmente en lo que concierne al desarrollo de la escritura, la comunicación y el aprendizaje de un idioma, el uso habitual de las TIC debe destacarse por ir más allá de su utilidad instrumental (Gros, 2000). De modo que la integración de las TIC debe considerar el respeto y desarrollo de su lengua materna como eje central.

Lo anterior, depende, mayormente, del establecimiento de políticas educativas que sean capaces de responder de manera pertinente a las demandas del sector. Sin embargo, para el caso de América Latina, vale hacer referencia a lo expresado por Bladergroen et al. (2012), quienes indican que uno de los obstáculos para la integración significativa de las TIC en los países en desarrollo ha sido la desalineación entre la política educativa y su instrumentación. Es pues, necesario, que las políticas educativas en esta materia procuren la integración en el ámbito digital, el acceso universal, sostenible, ubicuo y asequible a las TIC para todos, incluyendo a los grupos menos privilegiados, marginalizados y vulnerables, así como a los pueblos indígenas, $\mathrm{y}$, por tanto, facilitar la accesibilidad de las TIC para todos, en el marco del acceso a la información y al conocimiento.(Conferencia Mundial de Desarrollo de las Telecomunicaciones - Estambul, UIT, 2002).

\section{PROCEDIMIENTO METODOLÓGICO}

Con el propósito de obtener información relevante en relación con el uso de las TIC para la enseñanza de lenguas indígenas en la región latinoamericana, se llevó a cabo una indagación exploratoria previa sobre la temática. Este tipo de investigación "se efectúa sobre un tema u objeto desconocido o poco estudiado, por lo que sus resultados constituyen una visión aproximada de dicho objeto, es decir, un nivel superficial de conocimientos" (Arias, 2012, p. 23).

Dicha indagación "no sigue esquemas fijos o invariables, sino que será el propio investigador el encargado de desarrollar una estrategia de información que satisfaga sus dudas acerca de qué o cómo estudiar algo" (Perelló, 2011, p. 76). Puede considerar, entre otras acciones, revisión bibliográfica relacionada con el tema, entrevistas de carácter informativo, análisis de casos concretos.

Para captar información de docentes distribuidos en la región, se optó por el diseño, validación y puesta en línea de un cuestionario que se dirigió a aquellos que enseñan lenguas indígenas en América Latina. Para su difusión en la Web se contactó a distintos organismos, instituciones, asociaciones y personas vinculadas con el tema. Tras su publicación por diez (10) semanas consecutivas, el cuestionario fue respondido por setenta y seis (76) docentes distribuidos en los siguientes países: Argentina, Chile, Bolivia, Perú, México, Ecuador, Colombia, Costa Rica y Guatemala.

El cuestionario se estructuró en doce (12) preguntas con las cuales se pretendió conocer información sobre: grado de instrucción o título obtenido, país de residencia, sexo, edad, lengua(s) indígena(s) que ha enseñado, niveles educativos en los cuales las ha enseñado, estrategias apoyadas en las TIC y dispositivos empleados por los docentes. También se incluyó una escala opinática con los siguientes aspectos: uso de las TIC para la enseñanza de lenguas indígenas, capacitación de docentes y estudiantes en el uso de las TIC, conectividad a Internet, validación de contenidos de materiales didácticos multimedia por parte de los nativos, presencia y promoción de la cosmovisión y cultura indígena en los materiales didácticos multimedia que se utilizan. Finalmente se solicita a los docentes su apreciación general con respecto al uso de las TIC para la enseñanza de lenguas indígenas. 
Por otro lado, se realizó un proceso de búsqueda de algunas propuestas educativas recientes para la enseñanza de lenguas indígenas apoyadas en las TIC disponibles en la Web, considerando como criterios básicos: propuestas auspiciadas por organismos o instituciones oficiales o privadas e iniciativas desarrolladas por nativos. Para ello se ingresó a los campos de búsqueda de: Google (general y de noticias), Youtube, Facebook, Twitter, Instagran.

Una vez obtenida la información, tanto de docentes como de búsqueda en la Web, se procedió a su tabulación y análisis.

\section{RESULTADOS Y DISCUSIÓN}

Atendiendo al procedimiento metodológico implementado en el estudio, los resultados obtenidos se presentan en dos grandes rubros a saber: apreciaciones de docentes encuestados y descripción de propuestas educativas recientes para la enseñanza de lenguas indígenas apoyadas en las TIC disponibles en la Web.

Al especificar el grado de instrucción y/o título obtenido los docentes encuestados, los mismos quedaron distribuidos de la siguiente manera: Licenciados en Pedagogía 20\%, 3er nivel $13 \%$, Máster 29\%, Grado en Educación Inicial 5\%, Profesor en Ciencias de la Educación 7\%, Licenciados en Educación Primaria $18 \%$, en curso $8 \%$. Como se puede observar, los docentes, en su gran mayoría, se ubican en un grado de instrucción profesional en el área de educación, algunos de ellos con estudios más avanzados a nivel de maestría. No obstante, otros manifiestan estar realizando estudios para la profesionalización docente; en este caso cabe mencionar que en países como Chile, por ejemplo, existe la figura del educador tradicional quien hace las veces de agente educativo que transmite los saberes y conocimientos indígenas en las escuelas; para desempeñarse como tal, debe contar con el aval de su su comunidad y, además recibe formación a través de diplomados o cursos de extensión.

En cuanto al sexo de los docentes, $50 \%$ se ubican en el sexo masculino, 33,3\% en el renglón femenino, mientras que un $16,7 \%$ prefirió no indicarlo. Sobre este particular puede inferirse que en materia de educación, continúa siendo un desafío, la inclusión de las mujeres indígenas en el ámbito laboral, especialmente en labores docentes para la enseñanza de la lengua materna. En este caso, aspectos como el monolingüismo, la dedicación exclusiva a tareas domésticas y la subordinación racial, siguen representando obstáculos importantes para la adquisición de destrezas educativas. (Bonder, 1994).
Con respecto a la edad de los docentes, la gran mayoría, con un $50 \%$, son mayores de 51 años, el resto se distribuye en edades que oscilan entre 31 y 50 años. En este renglón llama poderosamente la atención la ausencia de jóvenes como transmisores formales de las lenguas indígenas; esto de alguna forma concuerda con el hecho de que son muchos los jóvenes indígenas que se sienten vulnerados al hablar su idioma nativo. (Rodríguez, 2015)

En relación con las lenguas que enseñan, resultaron con mayor porcentaje: Quechua (36\%) y Nahuatl (33\%), seguidas por Aimara (13\%). Se ubican con un porcentaje menor (de 3\% a 5\%), las siguientes lenguas: Kichwa, Malecu, Piapoco, Achí, Mapudungun. Tales cifras permiten afirmar que países como Bolivia, Perú y México, cuentan con políticas educativas que de algún modo motivan la enseñanza de sus lenguas indígenas dada la cantidad de docentes responsables de ello, en comparación con el resto.

La enseñanza de lenguas indígenas por parte de los docentes encuestados se ubica, mayormente, en Educación Básica o Primaria (51\%), seguida por su enseñanza a nivel de Preescolar (25\%) y en ámbitos comunitarios de manera informal (13\%), la Educación Media, cuenta con $11 \%$. Los resultados obtenidos en este renglón coinciden con los lineamientos de algunos países como México, Bolivia, Perú y Chile, donde la enseñanza de lenguas indígenas forma parte del currículum educativo con mayor énfasis en preescolar y educación básica. En ámbitos comunitarios, en los cuales se incluyen las comunidades virtuales, la enseñanza de las lenguas indígenas, obedece, en mayor parte, a iniciativas propias de sus integrantes.

El uso de las TIC, por su parte, se distribuye porcentualmente de la siguiente forma: búsqueda de información disponible en Internet, con un 98\%, siendo una de las estrategias que mayormente emplean para la enseñanza de las lenguas; específicamente lo hacen para obtener imágenes de contenidos a tratar y vídeos para mostrar durante el proceso.

A ello le sigue el uso de redes sociales como Facebook, con un $80 \%$. Ello da cuenta de la conformación de comunidades virtuales para la enseñanza informal de las lenguas. Mientras que muy pocos utilizan las TIC para la creación colaborativa de contenidos en la Web, y otros tantos, lo hacen de manera individual, lo cual se denota con un $35 \%$ y $12 \%$, respectivamente.

En la escala de opinión, al menos un $88 \%$ de los encuestados coincidió en afirmar que la cosmovisión de mundo de la cultura indígena no se encuentra presente en los materiales digitales diseñados para la enseñanza de estas lenguas. A la par, un $75 \%$ de los encuestados, estuvo totalmente de acuerdo con los siguientes aspectos: el uso de las TIC para la enseñanza de las 
lenguas indígenas se ve afectado por los problemas de conectividad a Internet presentes en la región, es necesario capacitar a docentes y estudiantes para el uso didáctico de las TIC, y los nativos deben validar los contenidos de los materiales didácticos disponibles en la red.

Entre las opiniones vinculadas al empleo de las TIC en y para la enseñanza de lenguas indígenas, se reitera la necesidad de capacitación en el uso de estas herramientas y mejorar el acceso a Internet en muchas poblaciones; el acceso a los dispositivos necesarios también es una necesidad manifestada por los docentes, extensiva a los estudiantes.

Otras opiniones destacan las bondades de las TIC para apoyar y contextualizar los procesos curriculares inherentes a la enseñanza de las lenguas, y la necesidad de incrementar políticas tendientes al empleo de las TIC para responder las demandas propias de esta era digital en cuanto a enseñanza, difusión y enriquecimiento de las lenguas indígenas.

En relación con los materiales demandan que los mismos sean mucho más interactivos y que contemplen más temas de conocimientos ancestrales.

En lo que concierne a propuestas educativas recientes para la enseñanza de lenguas indígenas apoyadas en las TIC disponibles en la Web, se tiene que, en líneas generales el uso de las TIC con énfasis en lo digital y el trabajo colaborativo en red, se encuentra de manera incipiente en la región para el estímulo y preservación de la comunicación en sus idiomas nativos.

Existen portales auspiciados por los distintos gobiernos de la región, que si bien no tienen como propósito fundamental la enseñanza de lenguas indígenas, constituyen un medio para su difusión pudiendo con ello motivar su apropiación y aprendizaje. Entre ellos pueden mencionarse los siguientes:

a) En México, el Portal Ciudadano en Lenguas Originarias, disponible en: http://comunidades.edomex.gob.mx/, y el portal de la Dirección General de Educación Indígena, disponible en: http://www.cdi.gob.mx/dgei/,

b) Lenguas nativas y criollas de Colombia, disponible en: https://www.mincultura.gov.co/areas/poblaciones/APP-delenguas-nativas/Paginas/default.aspx, y el Portal de Lenguas de Colombia, disponible en: https://lenguasdecolombia.caroycuervo.gov.co/

c) Lenguas originarias del Perú, disponible en: http://www.minedu.gob.pe/campanias/lenguas-originarias-delperu.php,

d) Educación intercultural. Lengua y cultura de los pueblos originarios (Chile-incluye materiales curriculares oficiales para consulta), disponible en: http://peib.mineduc.cl/lenguasoriginarias-2/,

e) Portal del Instituto Plurinacional de Estudio de Lenguas y Culturas de Bolivia, disponible en: https://www.ipelc.gob.bo/.

También se incluyen en este apartado, resultados que tienen que ver con aplicaciones móviles recientemente difundidas para la enseñanza y el aprendizaje de lenguas indígenas. Algunas de ellas son:

a) Vamos a aprender "Nahuatl", "Mixteco" y "Purépecha", desarrolladas por Laboratorios de Ciudadanía Digital y Manuvo, en México;

b) "Actividades Infantiles", "Cuentos Mágicos de los Ancestros", "Cuentos Mágicos de los Ancestros 2" y "Objetos Escondidos", desarrolladas por Fundación Sura y Colombia Games,

c) "ArandukApp" desarrollada por la Secretaría de Políticas Lingüísticas del Paraguay.

Todos estos recursos pueden ser incorporados a los procesos didácticos, no obstante, su uso está condicionado al acceso del dispositivo móvil y a Internet.

Otro hallazgo se corresponde con material en formato de vídeo disponible en la Web. Tras la revisión realizada, se encontró que muchos de ellos están auspiciados por diversas instituciones (públicas y privadas), en su diseño y desarrollo intervienen informáticos, programadores, diseñadores gráficos, dibujantes, editores de audio, lingüistas, sociólogos y profesores bilingües. Los contenidos, en general, se relacionan con leyendas, cuentos, canciones. Atendiendo a sus descripciones y comentarios, muchos de ellos son utilizados como material de apoyo en encuentros didácticos.

Finalmente, se encuentran las comunidades y servicios de interacción virtual. Por ejemplo, están: @lenguasdechile, @ lenguascolombia, "Lenguas indígenas de México en la Web" / @LenguasWeb, Coordinación Nacional de Antropología INAH /@cnan_inah /.

Otros grupos en Facebook, tales como: "Conozcamos más nuestra lengua náhuatl Marcelino Hernández Beatriz", "Nahuatl / @ nahuatlpotosino", "Kimeltuwe, materiales de mapudungun/@kimeltuwe”, "Quechua / @Quechua”, entre otros. Estos grupos y sitios responden a iniciativas particulares $\mathrm{y}$ otras tantas son promovidas por entes públicos y/o privados, $\mathrm{y}$ muchas se han creado para la difusión de contenidos e informaciones vinculadas a las lenguas indígenas, otras tantas divulgan materiales y contenidos digitales que pueden ser útiles para la enseñanza y el aprendizaje; varias de ellas también sirven como espacios de reflexión y discusión sobre la 
contemporaneidad de cada lengua y el uso cotidiano de expresiones.

\section{CONCLUSIONES}

Con base en los hallazgos del estudio puede darse respuesta al objetivo que orientó su desarrollo: Conocer los problemas que afronta hoy la enseñanza de las lenguas indígenas mediadas por las Tecnologías de la Información y la Comunicación en América Latina. De esa manera, los problemas y desafíos detectados en esta indagación exploratoria, son los siguientes:

Si bien la enseñanza de las lenguas indígenas tiene su espacio en el curriculo de preescolar y educación básica en gran parte de los países de América Latina, otros tantos no han formalizado dicho contenido en los procesos de formación de estos niveles educativos. A este respecto, es importante considerar que el aprendizaje de los niños y niñas en edad escolar será más fructífero y enriquecedor en la medida en que las escuelas planteen estrategias específicas que faciliten la actuación y comunicación de los estudiantes en su propia lengua. De allí se desprenden los siguientes desafíos: formar profesionalmente a hablantes de estos idiomas, ya que como conocedores de su lengua y cultura tendrán la facilidad de actuar como docentes frente a estudiantes indígenas; esto, a su vez, conlleva otro reto que tiene que ver con la motivación hacia la profesión docente, por lo que las políticas educativas, en este sentido, deben contemplar el otorgamiento de beneficios sociales y económicos que respondan de manera real a dicha labor. Tal desafío también incluye la promoción del sentido de pertenencia e identidad en las mujeres y jóvenes indígenas en la región.

$\checkmark \quad$ En lo que concierne al uso de los servicios y herramientas de la Web, se aprecian diversos propósitos, tales como: Divulgación de contenidos en lenguas indígenas, espacios para la comunicación e interacción, otros tantos para enseñanza de las lenguas $\mathrm{y}$, solo algunos se utilizan para promover la reflexión sobre las mismas. El desafío, en este sentido, se corresponde con la posibilidad de sistematizar las manifestaciones y discusiones que resultan de las interacciones entre los hablantes en tales espacios, con miras a enriquecer el debate actual sobre el uso de las lenguas, así como la cosmovisión y saberes contemporáneos que las caracterizan.

Se denota, así mismo, propósitos encontrados en cuanto al uso de tecnologías interactivas, por cuanto se aprecia que algunas de ellas se limitan a la mera traducción literal de lenguas indígenas al español, mientras que muy pocas van más allá dando espacio al significado cultural o simbólico que conlleva cada lengua en particular. Se plantea, en consecuencia, el desafío de modificar los paradigmas bajo los cuales se propone y desarrolla la enseñanza de las lenguas indígenas, considerando el aporte de los nativos para el diseño y pertinencia de materiales didácticos en el tema.

$\checkmark \quad$ Tradicionalmente, la enseñanza de lenguas indígenas y su cultura, se corresponde con su folklorización, dejando de lado la cosmovisión y saberes de estos pueblos. Dicha realidad también se encontró en algunos materiales disponibles en la Web. Frente a ello, el desafío es promover el cambio de mirada hacia lo indígena como una cultura con conocimientos propios, que bien pueden ser útiles para responder a las demandas de la sociedad actual. Ello también implica, asumir el uso de las TIC como instrumentos emancipatorios y no de dominación cultural y social.

Finalmente, la exploración llevada a cabo, también arrojó ciertas interrogantes, tales como: a) ¿Qué papel juegan los hablantes?, b) ¿Cómo puede ampliarse el espacio de acción para aquellos pueblos indígenas que aspiran a estructurar e implementar sus propios sistemas educativos?, c) ¿Qué impacto tiene en los hablantes, y en la sociedad en general, la creación de esos sistemas educativos indígenas en los que el español pasa a ser una segunda lengua?. Se exponen estas preguntas con el propósito de aportar ideas para futuros estudios y propuestas de acción en la enseñanza de las lenguas indígenas en América Latina.

\section{REFERENCIAS}

Arias, F. (2015). El Proyecto de Investigación, introducción a la metodología científica. Caracas, Venezuela.

Bladergroen, M., Chigona, W., Bytheway, A., Cox, S., Dumas, C., y Van Zyl, I. (2012). Educator discourses on ICT in education: A critical analysis. International Journal of Education and Development using Information and Communication Technology. 8, (2), 107-119.

Bonder, G. (1994). Mujer y Educación en América Latina: hacia la igualdad de oportunidades. Revista Iberoamericana de Educación, Número 6, Género y Educación. Septiembre Diciembre 1994.

CEPAL (2015). Agenda digital para América Latina y el Caribe (eLAC2018).

Degawan, M. (2019) Lenguas indígenas, conocimientos, esperanzas. Correo de la UNESCO, un solo mundo voces múltiples. Disponible en: https://es.unesco.org/courier/2019$1 /$ lenguas-indigenas-conocimientos-y-esperanza 
Dos Reis Batista, M. (2008). Considerações acerca do intercultural no ensino do Português brasileiro para falantes de outras línguas: Por que e para quê?. Anais do II Fórum de Línguas Estrangeiras da Universidade Federal do Pará, Belem.

Godenzzi, J. (2001). Política lingüística y educación en el contexto latinoamericano: el caso del Perú. II Congreso Internacional de la Lengua Española, Valladolid (España),

Gross, B. (2000). El ordenador invisible, hacia la apropiación del ordenador en la enseñanza. Barcelona: Editorial Gedisa.

Guerrero, M. y Dote, F. (2011). Integración curricular de tic's en la enseñanza de lenguas indígenas en latinoamérica. Revista electrónica Diálogos Educativos. ISSN 0718-1310

Hinton. L (2001). The Green Book of Language Revitalization in Practice. San Diego: Academic Press.

Lagos, C. (2015). El Programa de Educación Intercultural Bilingüe y sus resultados: ¿perpetuando la discriminación?. Pensamiento Educativo. Revista de Investigación Educacional Latinoamericana 2015, 52(1), 84-94.

Moreno, F. (2006). La diversidad lingüística de Hispanoamérica: implicaciones sociales y políticas. Real Instituto Elcano.

OIT (1989). Convenio 169 de la Organización Internacional del Trabajo. Disponible en: http://www.ilo.org/dyn/normlex/es/f?p=NORMLEXPUB:12 100:0::NO::P12100_INSTRUMENT_ID:312314

ONU (1989). Convención sobre los Derechos del Niño, adoptada por la Asamblea General de la Organización de las Naciones Unidas el 20 de Noviembre de 1989

ONU (2007). Declaración de las Naciones Unidas sobre los derechos de los pueblos indígenas.

ONU (2015). Resolución A/RES/70/1 Transformar nuestro mundo: la Agenda 2030 para el Desarrollo Sostenible, 25 de noviembre de 2015. [edición electrónica] http://www.un.org/es/comun/docs/?symbol=A/RES/70/1.

ONU (2019a). Foro Permanente para las Cuestiones Indígenas Informe sobre el $18^{\circ}$ período de sesiones ( 22 de abril de a 3 de mayo de 2019). Consejo Económico y Social Documentos Oficiales, Suplemento núm. 23

ONU (2019b). Foro Permanente para las Cuestiones Indígenas. Documentos antecedentes. Lenguas Indígenas. Disponible en:

https://www.un.org/es/events/indigenousday/assets/pdf/Bac kgrounder-Languages-Spanish\%202019.pdf

Perelló, S. (2011). Metodología de la Investigación Social.

Rodríguez, E. (2015). Juventudes latinoamericanas: prácticas socioculturales, políticas y políticas públicas
Rotaetxe, K. (1990). Sociolingüística. Madrid: Síntesis

Sánchez, J. (2008) Integración curricular de la TICs: conceptos e ideas. www.c5.cl/mici/pag/papers/inegr_curr.pd

Sordo, J. (2017). Adquisición escolar del español y minusvaloración de las propias competencias lingüísticas en estudiantes indígenas emigrados a Nuevo León. Revista mexicana de investigación educativa, vol. 22, núm. 75, 2017. Consejo Mexicano de Investigación Educativa A.C.

UNESCO (2010). Atlas de las Lenguas del Mundo en Peligro, [en línea] Ediciones UNESCO. París. [citado 30-09-2013] http://www.unesco.org/culture/languagesatlas/es/atlasmap.html

UNESCO (2016).Recomendaciones de Políticas Educativas en América Latina en base al TERCE. Oficina Regional de Educación para América Latina y el Caribe, OREALC/ UNESCO Santiago.

UNESCO (2017). Conocimiento indígena y políticas educativas en América Latina: análisis exploratorio de cómo las cosmovisiones y conceptos culturales indígenas de conocimiento inciden, y pueden incidir, en la política educativa en la región. https://unesdoc.unesco.org/ark:/48223/pf0000247754 